Christian Larsen

The $Q(s, S)$ control policy for the joint replenishment problem extended to the case of correlation among item-demands

Logistics/SCM

Research Group 


\title{
The $Q(s, S)$ control policy for the joint replenishment problem extended to the case of correlation among item-demands
}

\author{
Christian Larsen \\ Department of Business Studies \\ Logistics/SCM Research Group \\ Aarhus School of Business \\ University of Aarhus \\ Fuglesangs Allé 4 \\ DK-8210 Aarhus V
}

Denmark

\begin{abstract}
We develop an algorithm to compute an optimal $Q(s, S)$ policy for the joint replenishment problem when demands follow a compound correlated Poisson process. It is a non-trivial generalization of the work by Nielsen and Larsen (2005). We make some numerical analyses on two-item problems where we compare the optimal $Q(s, S)$ policy to the optimal uncoordinated $(s, S)$ policies. The results indicate that the more negative the correlation the less advantageous it is to coordinate. Therefore, in some cases the degree of correlation determines whether to apply the coordinated $Q(s, S)$ policy or the uncoordinated $(s, S)$ policies. Finally, we compare the $Q(s, S)$ policy and the closely connected $P(s, S)$ policy. Here we explain why the $Q(s, S)$ policy is a better choice if itemdemands are correlated.
\end{abstract}

Keywords joint replenishment problem, compound correlated Poisson process

\section{Introduction}

The joint replenishment problem concerns a multi-item inventory control problem where the replenishment cost structure is as follows. Each time a replenishment order is made, an item-specific replenishment cost $a_{k}$ is incurred for each item $k, k=1,2 . ., K$, in the replenishment order. In addition, a joint replenishment cost, $A$, is also incurred irrespective of the number of items included in the replenishment order. But, of course, at least one item must be included. It is also assumed that each item $k$ on inventory leads to an inventory holding cost at rate $h_{k}$ per item. We assume that all unfilled demand is backlogged. Each item $k$ on the backlog list incurs a penalty cost at rate $p_{k}$ per item. Furthermore, when a unit of item $k$ is put on the backlog list, a fixed penalty $\operatorname{cost} \pi_{k}$ per item is incurred. If a replenishment order is issued for item $k$, it has a fixed lead-time $L_{k}$. The joint replenishment problem has received considerable attention in the literature. One stream of research assumes that the demands are deterministic, cf Visvanathan (1996) and Wildeman et al (1997). Another stream assumes that demands follow a stochastic process, where the focus has been on analyzing good control policies. The can order 
policy is a prominent example. It was first analyzed by Balinfy (1964) and subsequently by Silver (1981), Federgruen, Groenevelt and Tijms (1984), Schultz and Johansen (1999), Melchiors (2002) and Johansen and Melchiors (2003). The main problem with the can order policy is to develop a valid mathematical model. Others have proposed policies where the coordination of replenishment decisions is secured by only allowing replenishments at certain time points. This is accomplished by either having deterministic review intervals or a common stochastic review interval governed by the total demand since the last replenishment opportunity. Atkins and Iyogun (1988) developed base-stock policies based on deterministic review intervals. If instead letting the review interval depend on the total demand since last replenishment opportunity the $Q S$ policy proposed by Reenberg and Planche (1967) is obtained. It was subsequently analyzed by Pantumsinchai (1992). Viswanathan (1997) formulated a $P(s, S)$ policy, where there is a common deterministic review interval and the replenishment decision of each item is governed by an $(s, S)$ policy. He also proposed a generalization of the $P(s, S)$ policy, which he called the $Q(s, S)$ policy, where the review interval is stochastic as described above. For this policy, Nielsen and Larsen (2005) have developed a mathematical model and an algorithm to compute optimal policy variables.

All the studies mentioned under the stochastic stream of research assume that itemdemands are independent stochastic processes, almost always Poisson processes. In this paper we relax on this assumption. We still assume that customers arrive after a Poisson process (with intensity $\lambda$ ) but demands for the items $k=1,2, \ldots K$ is specified by a $K^{\prime}$ th dimensional, non-negative, integer-valued random variable $\boldsymbol{X}$ with a given probability distribution $\mathrm{P}(\boldsymbol{X}=\boldsymbol{x})$. This means that item-demands are correlated. We denote this process a compound correlated Poisson process. Ohno and Ishigaki (2001) have also studied the joint replenishment problem under this demand process. They developed a modified policy iteration algorithm to find an optimal policy. This optimal policy will most often have no structure, see Ignall (1969) and Ohno and Ishigaki (2001) for numerical examples, and therefore it will be very difficult to implement in practice. Though correlation is present in their model, it seems to be unnoticed by Ohno and Ishigaki (2001). They do not look into ways in which correlation affects the optimal policy and the minimum costs. Contrary to them we will focus on an operational control policy, namely the $Q(s, S)$ policy, and also make sensitivity analyses with respect to correlation. We develop an algorithm to compute an optimal $Q(s, S)$ policy for a compound correlated Poisson process. It is a non-trivial generalization of the work by Nielsen and Larsen (2005). Since not very many have analyzed inventory control problems in the presence of correlated demands this should be considered as the first main contribution of our paper. The second main contribution is more qualitatively motivated and is illustrated by numerical examples. It concerns the following three questions. What is the impact of correlation on cost performance; is it worthwhile to coordinate; and if coordinating - how much information should be passed on to the subunits in charge of the inventory control of each item. In the numerical examples we consider two-item problems. We always let the marginal item-demand probability distributions be fixed. But we let the correlation vary enabling us to make systematic sensitivity analyses. The first two questions are investigated simultaneously. Here we demonstrate that the more negative the correlation the higher the cost rate of the optimal 
$Q(s, S)$ policy. We also compare the performance of the optimal $Q(s, S)$ policy to a decentralized setting where no attempt of coordinating the replenishment decisions are made. It means that for each item we find the optimal continuous review $(s, S)$ policy thus assuming that each item $k$ is managed independently having a replenishment cost $a_{k}+A$. Per definition, the optimal uncoordinated $(s, S)$ policies are insensitive to the correlation, because the cost structure is separable. Therefore, when taking previous observations into account, it is possible to find cases where the optimal decentralized policy will perform better than the coordinated optimal $Q(s, S)$ policy if the correlation is negative and the opposite holds if the correlation is positive. We find such cases interesting, because it underlines the importance of examining the demand pattern for correlation before considering whether to coordinate or not. When examining the last qualitative question we compare the $Q(s, S)$ policy to the closely connected $P(s, S)$ policy. Through a numerical example we explain and illustrate why the $Q(s, S)$ policy is better suited than the $P(s, S)$ policy for cases of correlated item-demands.

In Section 2 we develop our mathematical model and explain our algorithm. In Section 3 we investigate the first two qualitative questions. Section 4 deals with the last qualitative question. Finally, Section 5 contains some concluding remarks.

\section{Computation of an optimal $Q(s, S)$ policy}

In this section we explicitly assume that $P\left(X=\mathbf{0}_{\boldsymbol{K}}\right)=0$, where $\boldsymbol{0}_{\boldsymbol{K}}$ is the $K$ 'th dimensional vector consisting of zeros. Due to basic theory about Poisson processes, this contains no limitations in the assumptions made - see Tijms (1994; pp 21-22). Specifically a compound correlated Poisson process with rate $\lambda$ and probability distribution $P(\boldsymbol{X}=\boldsymbol{x})$ with $P\left(\boldsymbol{X}=\boldsymbol{0}_{\boldsymbol{K}}\right)>0$ is equivalent to a Poisson process with rate $\left(1-P\left(X=\boldsymbol{0}_{\boldsymbol{K}}\right)\right) \lambda$ and probability distribution $P(\boldsymbol{X}=\boldsymbol{x}) /\left(1-P\left(X=\boldsymbol{0}_{\boldsymbol{K}}\right)\right)$ for all vectors $\boldsymbol{x}$ with at least one positive element, while the probability of $\boldsymbol{0}_{K}$ is zero.

\subsection{The decomposition approach}

Consider a specific item $s p$, and let $Q$ be fixed. Assume that the joint replenishment cost $A$ is incurred when the aggregate demand since last replenishment opportunity is greater than or equal to $Q$. Then the optimal control policy (which is of type $(s, S)$ ) of item $s p$ should be computed under the assumption that item $s p$ only bears the item-specific replenishment cost $a_{s p}$. Define the random variable $X_{o t}=\sum_{k \neq s p} X_{k}$. It is the total demand per customer of all other (therefore the abbreviation ot) items than item $s p$. Given the probability distribution of $\boldsymbol{X}$, it is straightforward to specify the joint probability of $\left(X_{s p}\right.$, $\left.X_{o t}\right)$ by $p(u, v)=P\left(X_{s p}=u, X_{o t}=v\right)$. In order to have a finite state space we also assume there are upper bounds $U_{s p}, U_{o t}$ on the two random variables respectively. Define $X_{t o t}=$ $X_{s p}+X_{o t}$. Define $N_{t o t}=\min \left\{n: \sum_{i=1}^{n} X_{t o t}^{i} \geq Q\right\}$ where $X_{t o t}^{i} i=1,2, \ldots, n$ are identical and 
independent random variables having the same distribution as $X_{\text {tot }}$. Let $N_{\text {tot }}$ be the total number of customers appearing between two consecutive replenishment opportunities. Therefore for any given $Q$ the long run average costs per time unit (in the following often just denoted average costs) of the optimal $Q(s, S)$ policy is evaluated as

$$
C(Q)=\frac{\lambda A}{E\left[N_{t o t}\right]}+\sum_{s p=1}^{K} g_{s p}^{*}(Q)
$$

where $g_{s p}^{*}(Q)=\min \left\{g_{s p}(Q, s, S): s<S\right\}$ is the average cost of the optimal $(s, S)$ policy for item $s p$ and $g_{s p}(Q, s, S)$ is the average cost of an arbitrary $(s, S)$ policy when the review interval is stochastic, governed by the variable $Q$. The event $\left\{N_{t o t}=q\right\}$ can be rewritten to

$$
\left\{N_{\text {tot }}=q\right\}=\left\{\sum_{i=1}^{q} X_{t o t}^{i} \geq Q\right\} \backslash\left\{\sum_{i=1}^{q-1} X_{t o t}^{i} \geq Q\right\}
$$

where the symbol $\backslash$ here denotes set subtraction. The latter is empty if $q=1$ and is a subset of the former if $q>1$. Therefore

$$
E\left[N_{t o t}\right]=Q-\sum_{q=1}^{Q-1} P\left(\sum_{i=1}^{q} X_{t o t}^{i} \geq Q\right)
$$

\subsection{Computation of the optimal $(s, S)$ policy for item $s p$}

Before proceeding we introduce some more notations. By $p^{n}(u, v)$ we denote $P\left(\sum_{i=1}^{n} X_{s p}^{i}=u, \sum_{i=1}^{n} X_{o t}^{i}=v\right)$ where $X_{s p}^{i} \quad i=1,2 \ldots, n$ are identical, independent random variables with the same distribution as $X_{s p}$ and where $X_{o t}^{i} i=1,2, \ldots, n$ are identical, independent variables with the same distribution as $X_{o t}$

It holds

$$
p^{n}(u, v)=\sum_{x=0}^{u} \sum_{w=0}^{v} p(x, w) p^{n-1}(u-x, v-w)
$$

As usual the inventory position of an item is the inventory on stock - the number of units on the backlog list plus the number of units on order but not yet arrived. Define the inventory system to be in state $(y, r)$ when the inventory position of item $s p$ is $y$ and the total demand since the last replenishment opportunity occurred is $r$.

Define 
$G(y)$ The expected total costs incurred until the total demand is at least $Q$ when starting in state $(y, 0)$.

$N(y)$ The expected total costs incurred until state $(S, 0)$ is reached when starting in state $(y, 0)$.

$M(j)$ The expected time until state $(S, 0)$ is reached when starting in state $(s+j, 0)$ when $j$ $>0$. We define $M(j)=0$ if $j \leq 0$.

It follows from renewal-reward theory, see Tijms (1994; Chapter 1), that

$$
g_{s p}(Q, s, S)=\frac{N(S)}{M(S-s)}
$$

The expression $\tilde{p}_{u}=\sum_{q=1}^{Q} P\left(\sum_{i=1}^{q} X_{s p}^{i}=u, N_{t o t}=q\right)$ is the probability that the total demand for the specific item is $u$ during the stochastic review period. In Nielsen and Larsen (2005) it was a binomial probability. Now it is more involved but can be computed as follows.

From (2) it holds that

$$
\begin{aligned}
& P\left(\sum_{i=1}^{q} X_{s p}^{i}=u, N_{t o t}=q\right) \\
& =P\left(\sum_{i=1}^{q} X_{s p}^{i}=u, \sum_{i=1}^{q} X_{t o t}^{i} \geq Q\right)-P\left(\sum_{i=1}^{q} X_{s p}^{i}=u, \sum_{i=1}^{q-1} X_{t o t}^{i} \geq Q\right) \\
& =P\left(\sum_{i=1}^{q} X_{s p}^{i}=u, \sum_{i=1}^{q} X_{o t}^{i} \geq Q-u\right)-\sum_{b=0}^{u} P\left(\sum_{i=1}^{q-1} X_{s p}^{i}=b, X_{s p}^{q}=u-b, \sum_{i=1}^{q-1} X_{o t}^{i} \geq Q-b\right) \\
& =P\left(\sum_{i=1}^{q} X_{s p}^{i}=u, \sum_{i=1}^{q} X_{o t}^{i} \geq Q-u\right)-\sum_{b=0}^{u} P\left(X_{s p}=u-b\right) P\left(\sum_{i=1}^{q-1} X_{s p}^{i}=b, \sum_{i=1}^{q-1} X_{o t}^{i} \geq Q-b\right)
\end{aligned}
$$

Note that the last term of (6) vanishes when $q=1$.

We now elaborate further on the numerator and the denominator of (5). We consider the latter first

For $j>0$ it holds that 
$M(j)=\frac{E\left[N_{t o t}\right]}{\lambda}+\sum_{u=0}^{j-1} \tilde{p}_{u} M(j-u) \quad j=1, \ldots, S-S$

The solution to (7) is, see Zheng and Federgruen (1991),

$M(j)=\frac{E\left[N_{t o t}\right]}{\lambda} \sum_{u=0}^{j-1} m(u) \quad j=1, \ldots, S-S$

where

$m(0)=\frac{1}{1-\tilde{p}_{0}}$

and

$m(j)=\frac{1}{1-\tilde{p}_{0}} \sum_{u=1}^{j} \tilde{p}_{u} m(j-u) \quad j=1,2, \ldots, S-S-1$

We now derive a computable expression for the numerator of (5). We follow a standard lead-time offsetting principle. Let the random variable $D_{s p}\left(L_{s p}\right)$ denote the aggregate demand of item $s p$ over a time interval of length $L_{s p}$. Its probability distribution can be computed by the recursion scheme in Adelson (1966). Assume that a demand instance has just occurred at time point $\hat{\tau}$ bringing the inventory position of item $s p$ at level $j$. By $\tilde{\tau}$ denote the time point of the next demand instance. Let $\gamma(j)$ be defined as the total expected inventory and penalty costs of item $s p$ incurred in the time interval $\left(\hat{\tau}+L_{s p}, \tilde{\tau}+L_{s p}\right]$. Following Tijms (1994; p. 238) (who quotes Federgruen and Schechner (1983)) it is given as

$$
\begin{aligned}
& \gamma(j)=\frac{h_{s p}}{\lambda} \sum_{v=0}^{j}(j-v) P\left(D_{s p}\left(L_{s p}\right)=v\right)+\frac{p_{s p}}{\lambda} \sum_{v=j}^{\infty}(v-j) P\left(D_{s p}\left(L_{s p}\right)=v\right) \\
& +\pi_{s p}\left[\sum_{v=0}^{j} P\left(D_{s p}\left(L_{s p}\right)=v\right) \sum_{u=j-v}^{\infty}(u-j+v) P\left(X_{s p}=u\right)+E\left[X_{s p}\right] P\left(D_{s p}\left(L_{s p}\right) \geq j+1\right)\right]
\end{aligned}
$$

Define

$c_{j, r}^{E}$ to be the total expected inventory and penalty costs incurred until next replenishment opportunity occurs, when a demand instance has just occurred bringing the inventory position of item $s p$ to level $j$ and the aggregate demand since the last replenishment opportunity occurred to level $r$. Then 
$c_{j, r}^{E}=\left\{\begin{array}{cc}\gamma(j)+\sum_{u=0}^{U_{s p}} \sum_{v=0}^{U_{o t}} p(u, v) c_{j-u, r+u+v}^{E} & r<Q \\ 0 & r \geq Q\end{array}\right.$

By a proof of induction, see Appendix A, it can be shown that

$c_{j, Q-v}^{E}=\gamma(j)+\sum_{u=0}^{v-1}\left[\sum_{n=1}^{v-1} P\left(\sum_{i=1}^{n} X_{s p}^{i}=u, \sum_{i=1}^{n} X_{o t}^{i} \leq v-1-u\right)\right] \gamma(j-u)$

Because $G(y)=c_{y, 0}^{E}$ we get

$G(y)=\gamma(y)+\sum_{u=0}^{Q-1}\left[\sum_{n=1}^{Q-1} P\left(\sum_{i=1}^{n} X_{s p}^{i}=u, \sum_{i=1}^{n} X_{o t}^{i} \leq Q-1-u\right)\right] \gamma(y-u) \quad y=s+1, \ldots, S$

It holds that

$$
N(y)=G(y)+a_{s p} \sum_{q=y-s}^{\infty} \tilde{p}_{q}+\sum_{q=0}^{y-s-1} \tilde{p}_{q} N(y-q) \quad y=s+1, \ldots, S
$$

The solution to (15) is, see Zheng and Federgruen (1991),

$$
N(y)=a_{s p}+\sum_{j=0}^{y-s-1} m(j) G(y-j) \quad y=s+1, \ldots, S
$$

Therefore, finding the optimal $(s, S)$ policy amounts to minimizing

$$
g_{s p}(Q, s, S)=\frac{a_{s p}+\sum_{j=0}^{S-s-1} m(j) G(S-j)}{\frac{E\left[N_{t o t}\right]}{\lambda} \sum_{j=0}^{S-s-1} m(j)}=\frac{\lambda}{E\left[N_{t o t}\right]} Z_{s p}(s, S)
$$


where $Z_{s p}(s, S)=\frac{a_{s p}+\sum_{j=0}^{S-s-1} m(j) G(S-j)}{\sum_{j=0}^{S-s-1} m(j)}$ can be minimized by the algorithm in Zheng and Federgruen (1991). Note that the probabilities, we need to compute in order to develop an algorithm, are of type $P\left(\sum_{i=1}^{n} X_{s p}^{i}=u, \sum_{i=1}^{n} X_{o t}^{i} \leq v\right)$. These can be computed given the joint distribution of $\left(X_{s p}, X_{o t}\right)$.

\subsection{Computing the optimal $Q$}

By combining the analyses of subsections 2.1 and 2.2 we get

$$
C(Q)=\frac{\lambda}{E\left[N_{t o t}\right]}\left(A+\sum_{s p=1}^{n} \min \left\{Z_{s p}(s, S): s<S\right\}\right)
$$

Therefore our algorithm to compute an optimal $Q(s, S)$ policy consists of two loops. An inner loop, with a given $Q$, where for each item we find the optimal $(s, S)$ policy by the algorithm in Zheng and Federgruen (1991), and an outer loop where $Q$ is varied over a sufficiently large interval. We have developed a computer code of this algorithm in Visual Basic.

\subsection{When is the approximation exact?}

The question concerns whether it is possible that all inventory positions are above the reorder levels $s_{k}, k=1, \ldots, K$ at the time of a review instance. This would imply that our mathematical model will unnecessarily assume a joint replenishment cost while no items trigger any replenishment. The more positive the probability of this event, the more pessimistically biased the cost expression (18) compared to the actual costs. We derive a simple condition to establish when this probability is zero. Let $I P_{k}$ be the inventory position of item $k$ immediately after the review, thus $s_{k}+1 \leq I P_{k} \leq S_{k}$. Let $q_{k}$ be the total demand of item $k$ during the next review period. Per definition, $\sum_{k=1}^{K} q_{k} \geq Q$. If no item $k$ triggers a replenishment it means that $q_{k}<I P_{k}-s_{k}$ holds for $k=1, \ldots, K$, and thus $\sum_{k=1}^{K} q_{k}<\sum_{k=1}^{K}\left(I P_{k}-S_{k}\right) \leq \sum_{k=1}^{K}\left(S_{k}-s_{k}\right)$. Therefore the probability under investigation is zero if $\sum_{k=1}^{K}\left(S_{k}-s_{k}\right) \leq Q$ 
This condition is easy to check. For instance, for the dataset of Table 1 in Nielsen and Larsen (2005) it can be seen that their computed policy fulfills (19).

\section{The $Q(s, S)$ policy versus no coordination}

In this section we focus on the impact of correlation on the cost of the optimal policy. Simultaneously we also examine the possible gains from coordinating versus not coordinating. In order to make correlation most meaningful we here only consider twoitem cases. Our investigation is numerically based and we consider both positive and negative correlation. Tables 1 and 2 state the values of $p(u, v)$ for cases of positive and negative correlation respectively.

$<$ Tables 1 and 2 about here $>$

For both cases the parameter $\Delta$ ranges from 0 to $2 / 9$. Note that we take care of that the demand rates of the items are constant irrespective of the value of $\Delta$. When $\Delta=0$, the two tables display $100 \%$ negative and positive correlation, respectively. When $\Delta=2 / 9$, the two tables are identical displaying the case of uncorrelated item-demand. However, the case $\Delta=2 / 9$ is not equivalent to the case when the demand process can be decomposed into of two independent compound Poisson processes. The latter, having marginal item distributions as of Tables 1 and 2, is illustrated in Table 3.

$<$ Table 3 about here $>$

As noted in the beginning of Section 2 , it is easy to modify the data of Tables 1 and 2 so that $P\left(X=\mathbf{0}_{\boldsymbol{K}}\right)=0$, making the demand data fit to our algorithm.

Throughout this section we have $h_{1}=h_{2}=2, p_{1}=p_{2}=4, \pi_{1}=\pi_{2}=30, L_{1}=L_{2}=2$.

In the first experiment we let $a_{1}=a_{2}=10$ and $A=30$. Our results are summarized in Table 4.

$<$ Table 4 about here $>$

Irrespective of the value of $\Delta$ the solution to the uncoordinated case remains the same having a cost rate 35.62 with $s=2$ and $S=10$ for both items. We see that the more negative the correlation the higher costs of the optimal $Q(s, S)$ and thus the advantage of coordination decreases. We also see that the difference is quite significant ranging from $4.30 \%$ to $11.06 \%$. In order to display a situation where the uncoordinated approach is preferable, we made a new experiment with $a_{1}=a_{2}=30$ and $A=10$. Because we let $a_{1}$ $+A=a_{2}+A$ stay the same, the solution to the uncoordinated case does not change. Our results are given in Table 5 . 
$<$ Table 5 about here $>$

Now we see that the uncoordinated approach is best for all cases of negative correlation as well as for the case of a small positive correlation. Thus the optimal choice between the coordinated $Q(s, S)$ policy and the uncoordinated $(s, S)$ policies depends on the parameter $\Delta$. When comparing the optimal policies in Tables 4 and 5, we always see a higher $Q$ in Table 5 than in Table 4. This might seem odd given the logic behind the decomposition approach and the fact that the joint replenishment cost is smaller for the cases in Table 5 than those in Table 4 . The reason is that almost always both items trigger a replenishment order simultaneously. Therefore the total replenishment cost is almost always 70 in the cases in Table 5 while it is almost always 50 for the similar cases in Table 4. Therefore it makes sense to have a (stochastically) larger review interval for the cases in Table 5 compared to the similar cases in Table 4. We also note that the cost figures reported in Tables 4 and 5 are exact, because (19) is fulfilled for all cases. We also want to emphasize that it is always possible to construct a $Q(s, S)$ policy that is marginally better than the uncoordinated $(s, S)$ policies, namely to let $Q=1$ and the values of $s$ and $S$ to remain unchanged. In this way in cases where both items simultaneously trigger a replenishment (which can happen because $P\left(X_{1}>0, X_{2}>0\right)>$ 0 ), the total replenishment costs can be reduced. However, our mathematical model can never compute an optimal policy with $Q=1$, due to the decomposition approach it applies.

\section{The $Q(s, S)$ policy versus the $P(s, S)$ policy}

Nielsen and Larsen (2005) have received some critical comments on the $Q(s, S)$ policy. Though clearly operational it might not make sense to a practitioner. The reason is that it will be necessary to count the total demand of all items, whose measures in principle could be very different. Of course, a simple way to remedy on the $Q(s, S)$ policy would be to rescale all demand data into monetary values and let the policy parameter $Q$ be expressed in monetary units as well. We will not pursue that possible "trapdoor" further. Instead we will make a comparison between the $Q(s, S)$ policy (in its pure form as stated in this paper) and the $P(s, S)$ policy in Vishwanathan (1997), which might be considered more practical than the $Q(s, S)$ policy. Both policies have almost the same form. The main distinction seems to be whether the review period is constant or stochastic. The same sort of decomposition approach is applied to develop an algorithm. It resembles a hierarchical decision making process, somewhat in the spirit of Dirickx and Jennergren (1979). A central authority decides when to replenish, and a subunit in charge of each item then decides how much of the item to replenish. For the $P(s, S)$ policy the information about the length of the review period $t$ is sufficient. Based on that information the subunit can decide on an optimal $(s, S)$ policy. For the $Q(s, S)$ policy the information contained in the policy variable $Q$ is obviously not sufficient. Here the subunit also needs information about the total demand process. Therefore the $Q(s, S)$ policy might be judged impractical because it requires more information to be collected and passed on to the subunits. 
However, when item-demands are correlated then the $P(s, S)$ policy suffers from a deficiency, because it simply cannot detect correlation (the same observation holds for the can order policy). We illustrate that feature using the numerical example in Section 3. When applying the methodology for computing the optimal $P(s, S)$ policy, much of the information in Tables 1 and 2 becomes redundant. One would have to let the arrival rates of each item be 1 , and the probability distribution of the item-demands be 0,1,2 with equal probability, ignoring any information about the joint probability distribution. We have made an implementation of the algorithm for computing the optimal $P(s, S)$ policy. The details for developing the algorithm are outlined in Appendix B. For the case where $a_{1}=a_{2}=10$ and $A=30$ we get that the optimal policy is $t=5.6$ and $(s, S)=(7,10)$ for both items with average costs 37.39. For the case where $a_{1}=a_{2}=30$ and $A=10$ we get that the optimal policy is $t=3.2$ and $(s, S)=(4,10)$ for both items with average costs 38.81. By use of Arena 9.0 we have also simulated, the two policies under the various compound correlated Poisson processes in Tables 1 and 2. These results are summarized in Tables 6 and 7.

$<$ Table 6 and 7 about here $>$

As can be seen the cost performance of the computed $P(s, S)$ policy depends on $\Delta$ though the mathematical model purports insensitivity. Similar to the findings when investigating the $Q(s, S)$ policy, the more positive the correlation the smaller the costs while the more negative the correlation the higher the costs. It is also interesting to note that for the case where $a_{1}=a_{2}=30$ and $A=10$, then the $P(s, S)$ policy is inferior to the uncoordinated solution for all the cases of Table 6 , while the opposite conclusion holds for the optimal $Q(s, S)$ policy, see Table 4 . Thus a different conclusion would have been reached if the only alternative to an uncoordinated solution was either a $Q(s, S)$ policy or a $P(s, S)$ policy. This is also reflected in that Table 6 shows some substantial differences between the costs of the optimal $Q(s, S)$ policies and the simulated costs of the $P(s, S)$ policy in favor of the $Q(s, S)$ policy. Our numerical results reveal that the cost prediction of the computed $P(s, S)$ policy is pessimistically biased compared to the actual costs. This bias is quite significant in Table 7. However this is due to the inaccuracy of the mathematical model of the $P(s, S)$ policy which assumes that at each review instance the joint replenishment cost is incurred. Obviously, for the case where $t=3.2$ and $(s, S)=(4,10)$ in Table 7 , it will imply that at some review instances the joint replenishment cost is unnecessarily incurred.

\section{Concluding remarks}

In this paper we have shown how to generalize the analysis in Nielsen and Larsen (2005) of the $Q(s, S)$ policy for the joint replenishment problem when demands follow a compound correlated Poisson process. Our numerical results indicate that the more negative the correlation the less advantageous it is to coordinate the replenishment decisions by use of the $Q(s, S)$ policy. These findings are also supported by some preliminary analyses by Brønmo (2005). The observation seems to hold for other coordinating policies, like those illustrated for the $P(s, S)$ policy in Tables 6 and 7. 
Therefore it is possible to construct cases where it depends on the degree of correlation between item-demands whether it is better to coordinate or to use uncoordinated $(s, S)$ policies. If the choice is to use a coordinated policy, it seems most appropriate to use one which is in fact capable of detecting correlation. In that respect the $P(s, S)$ policy (or a can order policy) is not a good choice. However, if the choice is to use uncoordinated $(s, S)$ policies or a coordinating policy ignoring correlation there are some spillovers in terms of an easier task of collection data, because only information about item demand probability distributions is needed. All in all the choice of policy depends on the degree of correlation (as well as how easy it is to collect data) and the magnitude of the joint replenishment cost. When policies for joint replenishment problems are discussed, it seems like the main concern is the magnitude of the joint replenishment cost - see for instance Silver et al (1998; p. 424). Our study shows that one might also pay attention to the aspect of correlation.

\section{References}

Adelson RM Compound Poisson distributions. Operational Research Quarterly Vol 17, 1966, pp 73-75.

Atkins DR, Iyogun PO Periodic versus “can order" policies for coordinated multi-item inventory systems. Management Science, Vol 34, 1988, pp 791-796.

Balinfy JL On a class of multi-item inventory problems. Management Science, Vol 10, 1964, pp 287-297.

Brønmo CS Aspects of the joint replenishment problem. PhD thesis, Aarhus School of Business, Denmark, 2005.

Dirickx YMI, Jennergren, LP Systems analysis by multi-level methods: with applications to economics and management. John Wiley, New York, 1979.

Federgruen A, Schechner Z Cost formulas for continous review inventory models with fixed delivery lags. Operations Research, Vol 31, 1983, pp 957-965.

Ignall, E Optimal continuous review policies for two product inventory systems with joint setup costs. Management Science, Vol 15, 1969, pp 278-283.

Johansen SG, Melchiors P Can-order policy for the periodic review joint replenishment problem. Journal of the Operational Research Society, Vol 54, 2003, pp 283-290.

Melchiors, P Calculating can-order policies for the joint replenishment problem by the compensation approach. European Journal of Operational Research, Vol 141, 2002, pp 587-595. 
Nielsen $\mathrm{C}$, Larsen $\mathrm{C}$ An analytical study of the $Q(s, S)$ policy applied to the joint replenishment problem. European Journal of Operational Research, Vol 163, 2005, pp 721-732.

Ohno K, Ishigaki T A multi-item continuous review inventory system with compound Poisson demand. Mathematical Methods of Operations Research, Vol 53, 2001, pp 147-165.

Pantumsinchai P A comparison of three joint ordering inventory policies. Decision Science, Vol 23, 1992, pp 111-127.

Renberg B, Planche R Un modèle pour la gestion simultanèe des $n$ articles d'un stock. Revue Francaise d'Informatique et de Recherche Opérationelle, Vol 6, 1969, pp 4759.

Rosling $\mathrm{K}$ Inventory cost rate functions with nonlinear shortage costs. Operations Research, Vol 50, 2002, pp 1007-1017.

Schultz H, Johansen SG Can-order policies for coordinated inventory replenishment with Erlang distributed times between ordering. European Journal of Operational Research, Vol 113, 1999, pp 30-41

Sliver EA, Pyke DF, Peterson R Inventory management and production planning and scheduling. John Wiley, New York (3'ed), 1998.

Tijms H Stochastic models. John Wiley, New York, 1994.

Viswanathan, S A new optimal algorithm for the joint replenishment problem. Journal of the Operational Research Society, Vol 47, 1996, pp 936-944.

Viswanathan, S Periodic review $(s, S)$ policies for joint replenishment inventory problems. Management Science, Vol 43, 1997, pp 1447-1454.

Wildeman RE, Frenk JBG, Dekker R An efficient optimal solution for the joint replenishment problem. European Journal of Operational Research, Vol 99, 1997, pp 433-444.

Zheng YS, Federgruen A Finding optimal $(s, S)$ policies is just as simple as evaluating a single policy. Operations Research, Vol 39, 1991, pp 654-665. 


\section{Appendix A}

\section{Proof of (13)}

First note that when $v=1$, then (13) coincides with (12). Now let variable $w>1$ and assume (13) is true for $v=1,2, . . w-1$. When this is combined with (12) and using $p(0,0)=$ 0 , it then holds that

$$
\begin{aligned}
& c_{j, Q-w}^{E}=\gamma(j)+\sum_{b=0}^{w-1} \sum_{c=0}^{w-b-1} p(b, c) c_{j-b, Q-w+b+c}^{E} \\
& =\gamma(j)+\sum_{b=0}^{w-1} \sum_{c=0}^{w-b-1} p(b, c) \gamma(j-b)+ \\
& \sum_{b=0}^{w-1} \sum_{c=0}^{w-1-b} p(b, c) \sum_{u=0}^{w-b-c-1} \gamma(j-b-u)\left[\sum_{n=1}^{w-b-c-1} P\left(\sum_{i=1}^{n} X_{s p}^{i}=u, \sum_{i=1}^{n} X_{o t}^{i} \leq w-b-c-1-u\right)\right] \\
& =\left[1+\sum_{c=1}^{w-1} p(0, c)+\sum_{c=1}^{w-1} p(0, c) \sum_{n=1}^{w-c-1} P\left(\sum_{i=1}^{n} X_{s p}^{i}=0, \sum_{i=1}^{n} X_{o t}^{i} \leq w-c-1\right)\right] \gamma(j) \\
& +\sum_{d=1}^{w-1}\left[\sum_{c=0}^{w-d-1} p(d, c)+\sum_{b=0}^{d} \sum_{c=0}^{w-1-d} p(b, c) \sum_{n=1}^{w-b-c-1} P\left(\sum_{i=1}^{n} X_{s p}^{i}=d-b, \sum_{i=1}^{n} X_{o t}^{i} \leq w-c-1-d\right)\right] \gamma(j-d) \\
& {\left[1+P\left(X_{s p}=0, X_{o t} \leq w-1\right)+\sum_{n=2}^{w-1} P\left(\sum_{i=1}^{n} X_{s p}^{i}=0, \sum_{i=1}^{n} X_{o t}^{i} \leq w-1\right)\right] \gamma(j)} \\
& +\sum_{d=1}^{w-1}\left[P\left(X_{s p}=d, X_{o t} \leq w-d-1\right)+\sum_{n=1}^{w-2} \sum_{b=0}^{d} \sum_{c=0}^{w-d-1} p(b, c) P\left(\sum_{i=1}^{n} X_{s p}^{i}=d-b, \sum_{i=1}^{n} X_{o t}^{i} \leq w-c-1-d\right)\right] \gamma(j-d) \\
& =\gamma(j)+\sum_{d=0}^{w-1}\left[\sum_{n=1}^{w-1} P\left(\sum_{i=1}^{n} X_{s p}^{i}=d, \sum_{i=1}^{n} X_{o t}^{i} \leq w-1-d\right)\right] \gamma(j-d)
\end{aligned}
$$

\section{Appendix B}

\section{Outline of the algorithm to compute an optimal $P(s, S)$ policy}

Following the decomposition approach in Vishwanathan (1997), for each item we need to compute an optimal $(s, S)$ policy for a given review interval $t$. For simplicity we skip all item indices. We assume that the demand of the item is specified as a compound Poisson 
process with rate $\lambda$ and the demand of each customer is specified by a positive integervalued random variable $X$. Our derivation of the cost expression follows that in Rosling (2002) (Model 3 therein). Let $t_{0}$ be the time point of a review instance and assume that the inventory position immediately after the replenishment decision is $x$. Let $V(x)$ denote the total inventory and backorder cost incurred in the time period from $t_{0}+L$ to $t_{0}+L+$ $t$. It is specified in the following way.

If $x \leq 0$

$V(x)=p(-x t+\lambda E[X] t(L+t / 2))+\pi \lambda t E[X]$

If $x>0$

$$
\begin{aligned}
V(x)= & p\left(-x t+\lambda E[X] t(L+t / 2)+(p+h) \sum_{y=0}^{x-1} \int_{0}^{t} P\left(D_{L+\tau} \leq y\right) d \tau\right. \\
& +\pi\left[\lambda t E[X]+\sum_{y=0}^{x-1}\left[P\left(D_{L+t} \leq y\right)-P\left(D_{L} \leq y\right)\right]\right]
\end{aligned}
$$

This corresponds to Rosling (2002; Expression 14). Note that an adaptation has to be made because Rosling (2002) use a review period of 1 while ours is $t$. The only complicating term in (B1b) is

$$
\sum_{y=0}^{x-1}(x-y) \int_{0}^{t} P\left(D_{L+\tau}=y\right) d \tau
$$

We describe two methods to compute it.

\section{Method 1}

Let $N_{B}$ be the total number of arrivals in a time interval of length $B$. That is, $N_{B}$ is Poisson distributed with mean $\lambda B$. Then the integral $\int_{0}^{t} P\left(D_{L+\tau}=y\right) d \tau$ can be evaluated as follows. If $y=0$

$\int_{0}^{t} P\left(D_{L+\tau}=y\right)=\frac{1}{\lambda}\left[P\left(N_{L} \leq 0\right)-P\left(N_{L+t} \leq 0\right)\right]$

If $y>0$

$$
\int_{0}^{t} P\left(D_{L+\tau}=y\right) d \tau=\frac{1}{\lambda} \sum_{m=1}^{y} P(X(m)=y)\left[P\left(N_{L} \leq m\right)-P\left(N_{L+t} \leq m\right)\right]
$$


Here $X(m)$ is the $m^{\prime}$ th fold convolution of $X$.

\section{Method 2}

Let $I(i, d)$ define the expected accumulated on-hand inventory in a time period of length $d$ when at the start of this time period the on-hand inventory is $i$. By conditioning on the first arrival (if any) during this time interval, we get

$$
I(i, d)=\left\{\begin{array}{cc}
0 & i \leq 0 \\
\int_{0}^{d}\left[i \tau+\sum_{j=1}^{\infty} P(X=j) I(i-j, d-\tau)\right] f(\tau) d \tau+i d \int_{d}^{\infty} f(\tau) d \tau & i>0
\end{array}\right.
$$

Here $f(\tau)$ is the density function of the exponential distribution with mean $1 / \lambda$. It can be rewritten to the following differential equation

$$
I(i, d)=\frac{i}{\lambda}\left(1-e^{-\lambda d}\right)+\lambda \sum_{j=1}^{i-1} P(X=j) \int_{0}^{d} H(i-j, d-\tau) e^{-\lambda \tau} d \tau
$$

It has solution

$$
I(i, d)=\frac{b_{0}^{i}\left(1-e^{-\lambda d}\right)}{\lambda}-e^{-\lambda d} \sum_{j=1}^{i-1} b_{j}^{i} d^{j}
$$

where the coefficients $b_{j}^{i}$ are given as

$$
b_{0}^{i}=i+\sum_{r=1}^{i-1} P(X=r) b_{0}^{i-r} \quad i=1,2, \ldots
$$

and

$$
b_{j}^{i}=\frac{1}{j} \sum_{r=1}^{i-j} P(X=r) b_{j-1}^{i-r} \quad j=1,2, . . i-1, i=1,2, \ldots
$$

We are now ready to evaluate (B2). When the total demand in the time period from $t_{0}$ to $t_{0}+L$ is $q$ then the net-inventory at time point $t_{0}+L$ is $x-q$. Therefore (B2) can be rewritten to

$$
\sum_{q=0}^{x-1} P\left(D_{L}=q\right) I(x-q, t)
$$




\section{Tables}

\begin{tabular}{|l|l|l|l|}
\hline$u \mid v$ & 0 & 1 & 2 \\
\hline 0 & $1 / 3-\Delta$ & $\Delta / 2$ & $\Delta / 2$ \\
\hline 1 & $\Delta / 2$ & $1 / 3-\Delta$ & $\Delta / 2$ \\
\hline 2 & $\Delta / 2$ & $\Delta / 2$ & $1 / 3-\Delta$ \\
\hline
\end{tabular}

Table 1 The values of $p(u, v)$ when positive correlation. $\lambda=1$.

\begin{tabular}{|l|l|l|l|}
\hline$u \mid v$ & 0 & 1 & 2 \\
\hline 0 & $\Delta / 2$ & $\Delta / 2$ & $1 / 3-\Delta$ \\
\hline 1 & $\Delta / 2$ & $1 / 3-\Delta$ & $\Delta / 2$ \\
\hline 2 & $1 / 3-\Delta$ & $\Delta / 2$ & $\Delta / 2$ \\
\hline
\end{tabular}

Table 2 The values of $p(u, v)$ when negative correlation $. \lambda=1$.

\begin{tabular}{|l|l|l|l|}
\hline$\left.u\right|_{v}$ & 0 & 1 & 2 \\
\hline 0 & $1 / 3$ & $1 / 6$ & $1 / 6$ \\
\hline 1 & $1 / 6$ & 0 & 0 \\
\hline 2 & $1 / 6$ & 0 & 0 \\
\hline
\end{tabular}

Table 3 The two independent compound Poisson processes where $\lambda=2$. 


\begin{tabular}{|l|l|l|l|l|l|}
\hline$\Delta$ & $Q$ & $C(Q)$ & $S$ & $S$ & Advantage to no coordination \\
\hline \multicolumn{6}{|c|}{ Positive correlation } \\
\hline 0 & $11-12$ & 31.68 & 7 & 9 & $11.06 \%$ \\
\hline 0.05 & 12 & 31.98 & 7 & 9 & $10.22 \%$ \\
\hline 0.1 & 12 & 32.30 & 7 & 9 & $9.32 \%$ \\
\hline 0.15 & 12 & 32.61 & 7 & 9 & $8.45 \%$ \\
\hline 0.2 & 12 & 32.91 & 7 & 9 & $7.61 \%$ \\
\hline \multicolumn{7}{|c|}{ No correlation } \\
\hline $2 / 9$ & 12 & 33.04 & 7 & 9 & $7.24 \%$ \\
\hline \multicolumn{7}{|c|}{ Negative correlation } \\
\hline 0.2 & 12 & 33.17 & 7 & 9 & $6.88 \%$ \\
\hline 0.15 & 12 & 33.44 & 7 & 9 & $6.12 \%$ \\
\hline 0.1 & 12 & 33.70 & 7 & 9 & $5.39 \%$ \\
\hline 0.05 & 11 & 33.91 & 7 & 9 & $4.80 \%$ \\
\hline 0 & $11-12$ & 34.09 & 7 & 9 & $4.30 \%$ \\
\hline
\end{tabular}

Table 4 Summary of results for the experiment with $a_{1}=a_{2}=10$ and $A=30$. The advantage to no coordination is relative to the costs of no coordination.

\begin{tabular}{|l|l|l|l|l|l|}
\hline$\Delta$ & $Q$ & $C(Q)$ & $S$ & $S$ & Advantage to no coordination \\
\hline \multicolumn{5}{|c|}{ Positive correlation } \\
\hline 0 & $15-16$ & 34.42 & 7 & 10 & $3.37 \%$ \\
\hline 0.05 & 15 & 34.81 & 7 & 10 & $2.27 \%$ \\
\hline 0.1 & 15 & 35.19 & 7 & 10 & $1.21 \%$ \\
\hline 0.15 & 14 & 35.52 & 6 & 10 & $0.28 \%$ \\
\hline 0.2 & 14 & 35.83 & 6 & 10 & $-0.59 \%$ \\
\hline \multicolumn{6}{|c|}{ No correlation } \\
\hline $2 / 9$ & 14 & 35.97 & 6 & 10 & $-0.98 \%$ \\
\hline \multicolumn{7}{|c|}{ Negative correlation } \\
\hline 0.2 & 14 & 36.10 & 6 & 10 & $-1.35 \%$ \\
\hline 0.15 & 14 & 36.37 & 6 & 10 & $-2.11 \%$ \\
\hline 0.1 & 14 & 36.64 & 6 & 10 & $-2.86 \%$ \\
\hline 0.05 & 14 & 36.87 & 6 & 10 & $-3.51 \%$ \\
\hline 0 & $13-14$ & 37.04 & 6 & 10 & $-3.99 \%$ \\
\hline
\end{tabular}

Table 5 Summary of results for the experiment with $a_{1}=a_{2}=30$ and $A=10$. The advantage to no coordination is measured relative to the costs of the no coordination. 


\begin{tabular}{|l|l|l|l|}
\hline$\Delta$ & $\begin{array}{l}\text { Simulated cost of } \\
\text { computed } P(s, S) \text { policy }\end{array}$ & $\begin{array}{l}\text { Advantage to computed } \\
\text { costs }\end{array}$ & $\begin{array}{l}\text { Advantage to optimal } Q(s, S) \\
\text { policy }\end{array}$ \\
\hline \multicolumn{5}{|c|}{ Positive correlation } \\
\hline 0 & $36.58(0.10)$ & $2.21 \%$ & $-13.40 \%$ \\
\hline 0.05 & $36.75(0.13)$ & $1.75 \%$ & $-12.98 \%$ \\
\hline 0.1 & $36.88(0.11)$ & $1.39 \%$ & $-12.42 \%$ \\
\hline 0.15 & $36.99(0.09)$ & $1.09 \%$ & $-11.84 \%$ \\
\hline 0.2 & $37.04(0.10)$ & $0.96 \%$ & $-11.15 \%$ \\
\hline \multicolumn{5}{|l|}{ No correlation } \\
\hline $2 / 9$ & $37.05(0.10)$ & $0.93 \%$ & $-10.82 \%$ \\
\hline \multicolumn{5}{|l|}{ Negative correlation } \\
\hline 0.2 & $37.08(0.10)$ & $0.85 \%$ & $-10.54 \%$ \\
\hline 0.15 & $37.13(0.07)$ & $0.71 \%$ & $-9.94 \%$ \\
\hline 0.1 & $37.18(0.08)$ & $0.57 \%$ & $-9.36 \%$ \\
\hline 0.05 & $37.18(0.08)$ & $0.57 \%$ & $-8.80 \%$ \\
\hline 0 & $37.19(0.10)$ & $0.55 \%$ & $-8.33 \%$ \\
\hline
\end{tabular}

Table 6 Analysis of the performance of the computed $P(s, S)$ policy in the case $a_{1}=a_{2}=$ $10, A=30$. For each combination of $\Delta$ and policy we made 20 simulations with a runlength of 40000 time units. The half width of the $95 \%$ confidence interval is stated in parenthesis. The advantage to computed costs is measured relative to the computed costs. The advantage to the $Q(s, S)$ policy is measured relative to the simulated costs.

\begin{tabular}{|l|l|l|l|}
\hline$\Delta$ & $\begin{array}{l}\text { Simulated cost of } \\
\text { computed } P(s, S) \text { policy }\end{array}$ & $\begin{array}{l}\text { Advantage to computed } \\
\text { costs }\end{array}$ & $\begin{array}{l}\text { Advantage to optimal } Q(s, S) \\
\text { policy }\end{array}$ \\
\hline \multicolumn{5}{|c|}{ Positive correlation } \\
\hline 0 & $36.90(0.10)$ & $5.18 \%$ & $-6.72 \%$ \\
\hline 0.05 & $37.28(0.11)$ & $4.15 \%$ & $-6.63 \%$ \\
\hline 0.1 & $37.39(0.11)$ & $3.85 \%$ & $-5.88 \%$ \\
\hline 0.15 & $37.48(0.12)$ & $3.60 \%$ & $-5.23 \%$ \\
\hline 0.2 & $37.53(0.12)$ & $3.47 \%$ & $-4.53 \%$ \\
\hline \multicolumn{5}{|l|}{ No correlation } \\
\hline $2 / 9$ & $37.53(0.10)$ & $3.47 \%$ & $-4.16 \%$ \\
\hline \multicolumn{5}{|l|}{ Negative correlation } \\
\hline 0.2 & $37.55(0.11)$ & $3.41 \%$ & $-3.86 \%$ \\
\hline 0.15 & $37.55(0.10)$ & $3.41 \%$ & $-3.14 \%$ \\
\hline 0.1 & $37.60(0.09)$ & $3.28 \%$ & $-2.55 \%$ \\
\hline 0.05 & $37.66(0.09)$ & $3.12 \%$ & $-2.10 \%$ \\
\hline 0 & $37.65(0.08)$ & $3.14 \%$ & $-1.62 \%$ \\
\hline
\end{tabular}

Table 7 Analysis of the performance of the computed $P(s, S)$ policy in the case $a_{1}=a_{2}=$ $30, A=10$. The same comments for Table 6 apply here. 


\section{Working Papers from Logistics/SCM Research Group}

L-2007-01 Christian Larsen: The $Q(s, S)$ control policy for the joint replenishment problem extended to the case of correlation among item-demands.

L-2006-11 Daniele Pretolani, Lars Relund Nielsen \& Kim Allan Andersen: A note on "Multicriteria adaptive paths in stochastic, time-varying networks".

L-2006-10 Lars Relund Nielsen, Kim Allan Andersen \& Daniele Pretolani: Bicriterion a priori route choice in stochastic time-dependent networks.

L-2006-09 Christian Larsen \& Gudrun P. Kiesmüller: Developing a closed-form cost expression for an $(R, s, n Q)$ policy where the demand process is compound generalized Erlang.

L-2006-08 Eduardo Uchoa, Ricardo Fukasawa, Jens Lysgaard, Artur Pessoa, Marcus Poggi de Aragão, Diogo Andrade: Robust Branch-Cut-and-Price for the Capacitated Minimum Spanning Tree Problem over a Large Extended Formulation.

L-2006-07 Geir Brønmo, Bjørn Nygreen \& Jens Lysgaard: Column generation approaches to ship scheduling with flexible cargo sizes.

L-2006-06 Adam N. Letchford, Jens Lysgaard \& Richard W. Eglese: A Branch-andCut Algorithm for the Capacitated Open Vehicle Routing Problem.

L-2006-05 Ole Mortensen \& Olga W. Lemoine: Business integration between manufacturing and transport-logistics firms.

L-2006-04 Christian H. Christiansen \& Jens Lysgaard: A column generation approach to the capacitated vehicle routing problem with stochastic demands.

L-2006-03 Christian Larsen: Computation of order and volume fill rates for a base stock inventory control system with heterogeneous demand to investigate which customer class gets the best service.

L-2006-02 Søren Glud Johansen \& Anders Thorstenson: Note: Optimal base-stock policy for the inventory system with periodic review, backorders and sequential lead times.

L-2006-01 Christian Larsen \& Anders Thorstenson: A comparison between the order and the volume fill rates for a base-stock inventory control system under a compound renewal demand process.

L-2005-02 Michael M. Sørensen: Polyhedral computations for the simple graph partitioning problem.

L-2005-01 Ole Mortensen: Transportkoncepter og IT-støtte: et undersøgelsesoplæg og nogle foreløbige resultater. 
L-2004-05 Lars Relund Nielsen, Daniele Pretolani \& Kim Allan Andersen: $K$ shortest paths in stochastic time-dependent networks.

L-2004-04 Lars Relund Nielsen, Daniele Pretolani \& Kim Allan Andersen: Finding the $K$ shortest hyperpaths using reoptimization.

L-2004-03 Søren Glud Johansen \& Anders Thorstenson: The $(r, q)$ policy for the lostsales inventory system when more than one order may be outstanding.

L-2004-02 Erland Hejn Nielsen: Streams of events and performance of queuing systems: The basic anatomy of arrival/departure processes, when focus is set on autocorrelation.

L-2004-01 Jens Lysgaard: Reachability cuts for the vehicle routing problem with time windows. 
ISBN 87-7882-183-5

Department of Business Studies

Aarhus School of Business

University of Aarhus

Fuglesangs Allé 4

DK-8210 Aarhus V - Denmark

Tel. +4589486688

Fax +4586150188

www.asb.dk 